\title{
A Statistical Analysis of Near Fault Earthquake: A Research Perspective
}

\author{
Rohan Majumder \\ Assistant Professor \\ Civil Engg. Dept. \\ Sir Padampat Singhania \\ University, Udaipur-313601 \\ Rajasthan, India
}

\author{
Prasun Chakrabarti \\ Professor \& Head \\ Dept. of Computer Sc.\& Engg. \\ Sir Padampat Singhania \\ University, Udaipur-313601 \\ Rajasthan, India
}

\author{
Abhirup Basu Roy \\ Chowdhury \\ Alumnus, Post Graduate Student \\ Nottingham Pavement Research \\ Centre \\ Department of Civil Engineering \\ University of Nottingham \\ Nottingham NG7 2RD, UK
}

\begin{abstract}
The authors have pointed out certain discovered facts related to statistical analysis of near fault earthquakes. Directivity effect occurs when the harmonic mean of the velocity of the fault rupture propagation towards a site varies from that of the shear velocity of the rock near the source by a constant factor. The geometric mean of the combined fling effects bears a relation among the individual geometric mean of strike-slip and dip-slip faults, the individual and total number of observed time stamps of both the slip faults. point estimation plays a pivotal role towards realizing the detrimental effect of near fault earthquake. If reduction of dynamic response is based upon single incidence of inelastic deformation and hypothesis of fairness is rejected, then for unit negative bias, the estimate of weight of the single parameter by either historical supervised or predictive means is represented as a complex variable.
\end{abstract}

\section{Keywords}

Near fault earthquake, directivity effect, fling effort, slip faults

\section{INTRODUCTION}

Directivity is an effect of a fault rupturing whereby earthquake ground motion in the direction of rupture propagation is more severe than that in other directions from the earthquake source. Ground motion is the movement of the earth's surface from earthquakes or explosions. Ground motion is produced by waves that are generated by sudden slip on a fault or sudden pressure at the explosive source and travel through the earth and along its surface. An earthquake is the shaking of the ground caused by an abrupt shift of rock along a fracture in the Earth, called a fault. The tectonic plates make up the slow-moving outer shield of Earth. The tectonic plates often stick due to friction. As pressure builds up from trying to move, the stress increases. When the stress overcomes the friction at the fault, an earthquake occurs, and the plates quickly thrust forward and release energy into the crust. Two recently proposed and closely related approximate methods to estimate the maximum lateral inelastic deformations demands of single-degree-of-freedom (SDOF) systems subjected to earthquake ground motions are investigated [1]. Reduction of dynamic response due to earthquake can be estimated based on dynamic neural network model. Dampers are used as specially designed devices in the structure to reduce dynamic response thereby improving seismic-resistant ability of the structure.

\section{STATISTICAL INTERPRETATION OF DIRECTIVITY EFFECT}

Directivity effect [2] occurs when the harmonic mean of the velocity of the fault rupture propagation towards a site varies from that of the shear velocity of the rock near the source by a constant factor.

Let $\mathrm{VF}_{\mathrm{t}}$ be the velocity of the fault rupture propagation towards a site at timing instant $\mathrm{t}$,

$\mathrm{c}$ be a constant factor

$\mathrm{SV}_{\mathrm{t}}$ be the shear velocity of the rock near the source and $\mathrm{n}$ be the number of time stamps of observation.

After the observation period, the harmonic mean of the velocity of the fault rupture propagation towards a site is represented by:

$$
\begin{aligned}
H V F_{t} & =\frac{n}{\sum_{t=1}^{n}\left(1 / V F_{t}\right)} \\
& =\frac{n}{\sum_{t=1}^{n}\left(1 / c . S V_{t}\right)}
\end{aligned}
$$

[by theoretical foundation]

$$
\begin{aligned}
& =\frac{n}{(1 / c) \cdot \sum_{t=1}^{n}\left[1 / S V_{t}\right]} \\
& =c \cdot\left[\frac{n}{\sum_{t=1}^{n} 1 / S V_{t}}\right]
\end{aligned}
$$

Hence, $\quad \boldsymbol{H V} \boldsymbol{F}_{\boldsymbol{t}}=\boldsymbol{c} \cdot \boldsymbol{H S V _ { t }}$

So, it is evident that "Directivity effect occurs when the harmonic mean of the velocity of the fault rupture propagation towards a site varies from that of the shear velocity of the rock near the source by a constant factor".

\section{STATISTICAL INTERPRETATION OF SLIP FAULTS}

An earthquake is the shaking of the ground caused by an abrupt shift of rock along a fracture in the Earth, called a fault. The tectonic plates make up the slow-moving outer shield of Earth. An earthquake is the shaking of the ground caused by an abrupt shift of rock along a fracture in the Earth, called a fault. The tectonic plates make up the slow-moving outer shield of Earth. The tectonic plates often stick due to friction. 
As pressure builds up from trying to move, the stress increases. When the stress overcomes the friction at the fault, an earthquake occurs, and the plates quickly thrust forward and release energy into the crust. The energy moving through the ground is what causes the shaking associated with an earthquake. Faults which move along the direction of the dip plane are dip-slip faults and described as either normal or reverse (thrust), depending on their motion. Faults which move horizontally are known as strike-slip faults and are classified as either right-lateral or left-lateral. The geometric mean of the combined fling effects bears a relation among the individual geometric mean of strike-slip and dip-slip faults, the individual and total number of observed time stamps of both the slip faults [3].

Let $\mathrm{x}_{1}$ be the attenuation reliability factor due to the orientation of strike-slip faults in the strike-parallel component of ground motion,

$\mathrm{y}_{2}$ be the attenuation reliability factor due to the orientation of dip-slip faults in the strike- normal component of ground motion,

$\mathrm{GM}\left(\mathrm{x}_{1}\right)$ be the geometric mean of the values of $\mathrm{x}_{1}$ observed at various $\mathrm{n} 1$ time stamps

$\mathrm{GM}\left(\mathrm{y}_{2}\right)$ be the geometric mean of the values of $\mathrm{y}_{2}$ observed at various time $\mathrm{n}_{2}$ stamps.

Let, $\mathrm{x}_{11}, \mathrm{x}_{12}, \ldots \ldots \ldots \mathrm{x}_{1} \mathrm{n}_{1}$ be the values of attenuation factor of strike-slip fault data set while $\mathrm{y}_{21}, \mathrm{y}_{22}, \ldots \ldots, \mathrm{y}_{2} \mathrm{n}_{2}$ be the values of attenuation factor of dip-slip fault data set. Then,

Similarly, $\begin{aligned} \text { or, } x_{11} \cdot x_{12} \ldots \ldots \ldots x_{1} n_{1} & =G M\left(x_{1}\right)^{n_{1}} \\ y_{21} \cdot y_{22} \ldots \ldots \ldots y_{2} n_{2} & =G M\left(y_{2}\right)^{n_{2}}\end{aligned}$

$$
G M(c)=\left(G M\left(x_{1}\right)^{n_{1}} \cdot G M\left(y_{2}\right)^{n_{2}}\right)^{1 /\left(n_{1}+n_{2}\right)}
$$

Hence, we can claim that "The geometric mean of the combined fling effects bears a relation among the individual geometric mean of strike-slip and dip-slip faults, the individual and total number of observed time stamps of both the slip faults".

\section{ANALYSIS OF DETRIMENTAL EFFECT}

Point estimation [4] plays a pivotal role towards realizing the detrimental effect of near fault earthquake. The near-fault earthquakes cause most of the seismic energy from the rupture to arrive in a single coherent long-period pulse of motion. The detrimental effect is to be sensed over a period of time and compared to the predicted one as per supervised learning rule. Point estimation is to be applied as the interval between two adjacent time stamps is negligible to estimate even minute fluctuation.

Case I: Detrimental effect is not matched in the observation period:

Let $\mathrm{X}(\mathrm{t})$ be the probability of no matching of detrimental effect of near fault earthquake in time $t$ and $X(t+\Delta t)$ will be that in time $(\mathrm{t}+\Delta \mathrm{t})$

Hence, $X(t+\Delta t)=X(t)[1-€ t]$, where $€$ is the constant of proportionality.

$\lim _{\Delta t \rightarrow 0} \frac{X(t+\Delta t)-X(t)}{\Delta t}=-€ X(\mathrm{t})$ or, proportionality

$$
\text { If } \quad \mathrm{t}=0, \mathrm{X}(\mathrm{t})=\mathrm{a}
$$

Case II: If only one or many detrimental effects is matched:

Let $X_{1}(t)$ be the probability that one effect is recognized successfully. So $X_{1}(t+\Delta t)$ is that in time $t_{2}$. It may happen that in time $t$ no matching occurs while it occurs in $\Delta t$. The reverse may also occur. Hence, $\mathrm{X}_{\mathrm{M}}(\mathrm{t})=\mathrm{X}_{\mathrm{M}}(\Delta \mathrm{t})=0.5=$ equally probable where $X_{M}(t)$ is probability of successful match in $t$ and $X_{M}(\Delta t)$ is that in $\Delta t$.

So,

$$
X_{1}(t+\Delta t)=X_{1}(t) \cdot(1-€ \Delta t)+X(t) € \Delta t
$$

Therefore, $X_{1}(t)=€ \mathrm{t} e^{-€ \mathrm{t}}+b e^{-€ \mathrm{t}}$, where ' $\mathrm{b}$ ' is another constant of proportionality.

If $\mathrm{b}=0$, then $X_{1}(t)=€ t e^{-€ t}$

Hence, if $n$ number of successful detrimental effect patterns are observed in time $t$, then,

$$
X_{n}(t)=\left((€ t)^{n} \cdot e^{-€ t} / n !\right.
$$

Hence point estimation plays a pivotal role towards realizing the detrimental effect of near fault earthquake.

\section{ANALYSIS OF INELASTIC DEFORMATION}

Two recently proposed and closely related approximate methods to estimate the maximum lateral inelastic deformations [5] demands of single-degree-of-freedom (SDOF) systems subjected to earthquake ground motions are investigated. In both methods, maximum inelastic displacements are estimated from maximum elastic displacements through the use of parameters that relate the response of elastic and inelastic SDOF systems. Analysis of inelastic deformation due to earthquake can be carried out in the light of Fuzzy estimation. Subjected to earthquake, the conventional structures are designed to resist ground motions through a combination of material strength, the structural elements' energy consuming ability, and overall structural deformability. During earthquakes, the structures are permitted to have significant inelastic deformation, which is designed to occur in critical regions of the structure, usually in the beam or near to beam-column joints. This significant inelastic behavior can absorb additional energy input by external ground motions. However, such permitted inelastic behavior also results in significant damage to the structure. In the view of above, we propose:

$$
x . €=1-\mathrm{y} £
$$

where $€$ and $£$ are fuzzy estimates of incident inelastic deformation and optimum permissible deformation in order to be seismic-resistant respectively, $\mathrm{x}$ be support factor of $€$ towards permissible expansion while y be the probabilistic support factor of $£$ reflecting the necessity of maintaining the tolerance limit.

\section{REDUCTION OF DYNAMIC RESPONSE}

Reduction of dynamic response [6] due to earthquake can be estimated based on dynamic neural network model. Dampers are used as specially designed devices in the structure to reduce dynamic response thereby improving seismic-resistant ability of the structure. The error in this context is the excess 
inelastic deformation and the target implies the optimum and permissible measure of reduction of dynamic response. For accurate forecasting for reduction of dynamic response, dynamic neural network model can be proposed in this perspective where error can be adjusted suing feedback path, neural network architecture can be modified and accordingly weights of the three most significant parameters viz. material strength of structure, structure elements' energy consuming ability and overall structural deformability can be adjusted.

If reduction of dynamic response is based upon single incidence of inelastic deformation and hypothesis of fairness is rejected, then for unit negative bias, the estimate of weight of the single parameter by either historical supervised or predictive means is represented as a complex variable.

\section{Justification:}

The proposed mathematical equation of neuro-fuzzy based reduction of dynamic response in case of any stochastic event is as follows:

$$
T_{p}+\beta=A W_{n}=\left[\left(\sum_{i=1}^{x} A W_{x, i} \cdot A W_{y, i}\right) / x\right]
$$

where,

$\mathrm{Tp}=$ average accuracy estimation of reduction of dynamic response,

$\beta=$ unit negative bias value

$\mathrm{AWn}=$ effective weight of the three most significant parameters viz. material strength of structure, structure elements' energy consuming ability and overall structural deformability leading to the seismic-resistant ability of the structure.

$\mathrm{AW}_{\mathrm{x}, \mathrm{i}}=$ estimate of weight of $\mathrm{i}^{\text {th }}$ parameter on the basis of sampled historical information based on supervised learning rule,

$\mathrm{AW}_{\mathrm{y}, \mathrm{i}}=$ estimate of weight of $\mathrm{i}^{\text {th }}$ parameter on the basis of present hypothesis

and, $\mathrm{x}=$ total number of instances of inelastic deformation.

As per our model, single incidence of inelastic deformation takes place and hypothesis of fairness is rejected.

Therefore, $\quad\left(\boldsymbol{A} \boldsymbol{W}_{\boldsymbol{x}, \boldsymbol{i}} \cdot \boldsymbol{A} \boldsymbol{W}_{\boldsymbol{y}, \boldsymbol{i}}\right)=\mathbf{0}+\boldsymbol{\beta}$

or, $\left(\mathrm{A} W_{y, 1}\right)^{2}=\beta$

$$
\begin{aligned}
& \text { or, }\left(\mathrm{A} W_{y, 1}\right)^{2}=-1[\text { since unit negative bias] } \\
& \text { or, }\left(\mathrm{A} W_{y, 1}\right)=(-1)^{1 / 2}
\end{aligned}
$$

Similarly, $\left(\mathrm{A} W_{x, 1}\right)=(-1)^{1 / 2}$

Hence it is justified to state that "If reduction of dynamic response is based upon single incidence of inelastic deformation and hypothesis of fairness is rejected, then for unit negative bias, the estimate of weight of the single parameter by either historical supervised or predictive means is represented as a complex variable".

\section{CONCLUSION}

The paper entails some discovered facts related to directivity effect of earthquakes, slip faults, detrimental effects, inelastic deformation, and reduction of dynamic response. The related statistical analysis has also been pointed out.

\section{REFERENCES}

[1] Indian Patent File number- 201711005075, Date of filing - 13/02/2017; Publication Date - 30/06/2017, TitleStatistical and Artificial Intelligence based analysis of vibration control of near fault earthquake; InventorsDr.Prasun Chakrabarti , Mr Rohan Majumder

[2] Somerville PG, Smith NF, Graves RW, Abrahamson NA "Abrahamson. Modification of empirical strong ground motion attenuation relations to include the amplitude and duration effects of rupture directivity". Seism. Res. Lett. 1997; 68: 199-222.

[3] K. Rybicki, "The Elastic Residual Field of a Very Long Strike Slip Fault in the Presence of a Discontinuity," Bulletin of the Seismological Society of America,1971: 61: 79-92

[4] Lagorio,H,J, EARTHQUAKES An Architect's Guide to Non-Structural Seismic Hazard, John Wiley \& Sons, Inc., USA ,1990

[5] Riddell, R., Garcia, J. E. and Garces, E., Inelastic deformation response of SDOF systems subjected to earthquakes. Earthquake Engng. Struct. Dyn., 2002: 31: 515-538. doi:10.1002/eqe.142

[6] Adam C. and Fotiu P.A., Dynamic response of earthquake excited inelastic primary-secondary systems, In Proc. 12 WCEE, 2000 